\title{
CHARACTERS OF PRIME DEGREE
}

\author{
EDITH ADAN-BANTE \\ Department of Mathematical Science, Northern Illinois University, Watson Hall 320, DeKalb, \\ IL60115-2888, USA \\ e-mail:EdithAdan@illinoisalumni.org
}

(Received 4 June 2008; accepted 26 May 2011)

\begin{abstract}
Let $G$ be a finite nilpotent group, $\chi$ and $\psi$ be irreducible complex characters of $G$ with prime degree. Assume that $\chi(1)=p$. Then, either the product $\chi \psi$ is a multiple of an irreducible character or $\chi \psi$ is the linear combination of at least $\frac{p+1}{2}$ distinct irreducible characters.
\end{abstract}

\section{Mathematics Subject Classification. 20c15}

1. Introduction. Let $G$ be a finite group and $\chi, \psi \in \operatorname{Irr}(G)$ be irreducible complex characters of $G$. We can check that the product $\chi \psi$ of $\chi$ and $\psi$, where $\chi \psi(g)=$ $\chi(g) \psi(g)$ for all $g$ in $G$, is a character and so it can be expressed as a linear combination of irreducible characters. Let $\eta(\chi \psi)$ be the number of distinct irreducible constituents of the product $\chi \psi$.

Theorem A. Let $G$ be a finite nilpotent group, $\chi$ and $\psi$ be irreducible complex characters of prime degree. Assume that $\chi(1)=p$. Then, one of the following holds:

(i) $\chi \psi$ is the sum of $p^{2}$ distinct linear characters.

(ii) $\chi \psi$ is the sum of $p$ distinct linear characters of $G$ and of $p-1$ distinct irreducible characters of $G$ with degree $p$.

(iii) all the irreducible constituents of $\chi \psi$ are of degree $p$. Also, either $\chi \psi$ is a multiple of an irreducible character, or it has at least $\frac{p+1}{2}$ distinct irreducible constituents and at most $p$ distinct irreducible constituents, i.e.

$$
\text { either } \eta(\chi \psi)=1 \text { or } \frac{p+1}{2} \leq \eta(\chi \psi) \leq p .
$$

(iv) $\chi \psi$ is an irreducible character.

It is proved in Theorem A of [1] that given any prime $p$, any $p$-group $P$, any faithful characters $\chi, \psi \in \operatorname{Irr}(P)$, either the product $\chi \psi$ is a multiple of an irreducible, or $\chi \psi$ is the linear combination of at least $\frac{p+1}{2}$ distinct irreducible characters, i.e. either $\eta(\chi \psi)=1$ or $\eta(\chi \psi) \geq \frac{p+1}{2}$. It is proved in [4] that given any prime $p$ and any integer $n>0$, there exists a $p$-group $P$ and characters $\varphi, \gamma \in \operatorname{Irr}(P)$ such that $\eta(\varphi \gamma)=n$. Thus, without the hypothesis that the characters in Theorem A of [1] are faithful, the result may not hold. In this note, we are proving that if the characters have 'small' degree then the values that $\eta(\chi \psi)$ can take have the same constraint as if they were faithful.

Present address: Department of Mathematics, University of Saint Thomas, 2115 Summit Avenue, Saint Paul, MN 55105-1079, USA 
2. Proofs. We are going to use the notation of [5]. In addition, we denote by $\operatorname{Lin}(G)=\{\chi \in \operatorname{Irr}(G) \mid \chi(1)=1\}$ the set of linear characters, and by $\operatorname{Irr}(G \bmod N)=$ $\{\chi \in \operatorname{Irr}(G) \mid \operatorname{Ker}(\chi) \geq N\}$ the set of irreducible characters of $G$ that contain in their kernel the subgroup $N$. Also, denote by $\bar{\chi}$ the complex conjugate of $\chi$, i.e. $\bar{\chi}(g)=\overline{\chi(g)}$ for all $g$ in $G$.

Lemma 2.1. Let $G$ be a finite group and $\chi, \psi \in \operatorname{Irr}(G)$. Let $\alpha_{1}, \alpha_{2}, \ldots, \alpha_{n}$, for some $n>0$, be the distinct irreducible constituents of the product $\chi \psi$ and $a_{1}, a_{2}, \ldots, a_{n}$ be the unique positive integers such that

$$
\chi \psi=\sum_{i=1}^{n} a_{i} \alpha_{i} .
$$

If $\alpha_{1}(1)=1$, then $\psi \overline{\alpha_{1}}=\bar{\chi}$. Hence, the distinct irreducible constituents of the character $\chi \bar{\chi}$ are $1_{G}, \overline{\alpha_{1}} \alpha_{2}, \overline{\alpha_{1}} \alpha_{2}, \ldots, \overline{\alpha_{1}} \alpha_{n}$, and

$$
\chi \bar{\chi}=a_{1} 1_{G}+\sum_{i=2}^{n} a_{i}\left(\overline{\alpha_{1}} \alpha_{i}\right) .
$$

Proof. See Lemma 4.1 of [3].

Lemma 2.2. Let $G$ be a finite p-group for some prime $p$ and $\chi \in \operatorname{Irr}(G)$ be a character of degree $p$. Then, one of the following holds:

(i) $\chi \bar{\chi}$ is the sum of $p^{2}$ distinct linear characters.

(ii) $\chi \bar{\chi}$ is the sum of $p$ distinct linear characters of $G$ and of $p-1$ distinct irreducible characters of $G$ with degree $p$.

Proof. See Lemma 5.1 of [2].

Lemma 2.3. Let $G$ be a finite p-group, for some prime $p$, and $\chi, \psi \in \operatorname{Irr}(G)$ be characters of degree $p$. Then, either $\eta(\chi \psi)=1$ or $\eta(\chi \psi) \geq \frac{p+1}{2}$.

Proof. Assume that the lemma is false. Let $G$ and $\chi, \psi \in \operatorname{Irr}(G)$ be a counterexample of the statement, i.e. $\chi(1)=\psi(1)=p$ and $1<\eta(\chi \psi)<\frac{p+1}{2}$.

Working with the group $G /(\operatorname{Ker}(\chi) \cap \operatorname{Ker}(\psi))$, by induction on the order of $G$, we may assume that $\operatorname{Ker}(\chi) \cap \operatorname{Ker}(\psi)=\{1\}$. Set $n=\eta(\chi \psi)$. Let $\theta_{i} \in \operatorname{Irr}(G)$, for $i=$ $1, \ldots, n$, be the distinct irreducible constituents of $\chi \psi$. Set

$$
\chi \psi=\sum_{i=1}^{n} m_{i} \theta_{i}
$$

where $m_{i}>0$ is the multiplicity of $\theta_{i}$ in $\chi \psi$.

If $\chi \psi$ has a linear constituent, then by Lemmas 2.1 and 2.2 we have that $\eta(\chi \psi) \geq p$. If $\chi \psi$ has an irreducible constituent of degree $p^{2}$, then $\chi \psi \in \operatorname{Irr}(G)$ and so $\eta(\chi \psi)=1$. Thus, we may assume that $\theta_{i}(1)=p$ for $i=1, \ldots, n$.

Since $G$ is a $p$-group, there must exist a subgroup $H$ and a linear character $\xi$ of $H$ such that $\xi^{G}=\chi$. Then, $|G: H|=\chi(1)=p$ and thus $H$ is a normal subgroup. By Clifford theory, we have then

$$
\chi_{H}=\sum_{i=1}^{p} \xi_{i}
$$


for some $\xi_{1}=\xi, \ldots, \xi_{p}$ distinct linear characters of $H$.

Claim 2.6. $H$ is an abelian group.

Proof. Suppose that $\psi_{H} \in \operatorname{Irr}(H)$. Since $\left(\xi \psi_{H}\right)^{G}=\chi \psi$ by Exercise 5.3 of [5], and $\xi \psi_{H} \in \operatorname{Irr}(H)$, it follows that either $\xi \psi_{H}$ induces irreducibly, and thus $\eta(\chi \psi)=1$, or $\xi \psi_{H}$ extends to $G$ and thus $\left(\xi \psi_{H}\right)^{G}$ is the sum of the $p$ distinct extensions of $\xi \psi_{H}$, i.e. $\eta(\chi \psi)=p$. Therefore, $\psi_{H} \notin \operatorname{Irr}(G)$ and since $H$ is normal in $G$ of index $p$ and $\psi(1)=p$, $\psi$ is induced from some $\tau \in \operatorname{Lin}(H)$.

Since both $\xi$ and $\tau$ are linear characters, we have that $\operatorname{Ker}(\xi) \cap \operatorname{Ker}(\tau) \geq[H, H]$. Observe that $\operatorname{core}_{G}(\operatorname{Ker}(\xi) \cap \operatorname{Ker}(\tau))=\operatorname{core}_{G}(\operatorname{Ker}(\xi)) \cap \operatorname{core}_{G}(\operatorname{Ker}(\tau))=\operatorname{Ker}(\chi) \cap$ $\operatorname{Ker}(\psi)$. Since $H$ is a normal subgroup of $G$, so is $[H, H]$ and thus $\{1\}=\operatorname{Ker}(\chi) \cap$ $\operatorname{Ker}(\psi) \geq[H, H]$. Therefore, $H$ is abelian.

By the previous claim, observe that $\psi$ is also induced by some linear character $\tau$ of $H$ and thus

$$
\psi_{H}=\sum_{i=1}^{p} \tau_{i}
$$

for some $\tau_{1}=\tau, \ldots, \tau_{p}$ distinct linear characters of $H$. Observe also that the centre of both $\chi$ and $\psi$ is contained in $H$.

Claim 2.8. $\mathbf{Z}(G)=\mathbf{Z}(\chi)=\mathbf{Z}(\psi)$.

Proof. Suppose that $\mathbf{Z}(\chi) \neq \mathbf{Z}(\psi)$. Set $U=\mathbf{Z}(\chi) \cap \mathbf{Z}(\psi)$. Either $U$ is properly contained in $\mathbf{Z}(\chi)$, or it is properly contained in $\mathbf{Z}(\psi)$. We may assume that $U<\mathbf{Z}(\psi)$ and thus we may find a subgroup $T \leq \mathbf{Z}(\psi)$ such that $T / U$ is chief factor of $G$. Since $H$ is abelian, $\mathbf{Z}(\psi)<H$ and $\tau^{\bar{G}}=\psi$, then $\psi_{T}=p \tau_{T}$ and so $\left(\tau_{i}\right)_{T}=\tau_{T}$ for $i=1, \ldots, p$. Because $\xi^{G}=\chi, \xi \in \operatorname{Lin}(H)$ and $T \not \leq \mathbf{Z}(\chi)$, the stabilizer of $\xi_{T}$ is $H$. Thus, the stabilizer of $\xi_{T} \tau_{T}$ in $G$ is $H$. By Clifford theory, we have that $\xi \tau_{i} \in \operatorname{Lin}(H)$ induces irreducibly and $\xi \tau_{i}$ are distinct characters for $i=1, \ldots, p$. By (2.7), we have that $\chi \psi=\left(\xi \psi_{H}\right)^{G}=\left(\xi\left(\tau_{1}+\cdots+\tau_{p}\right)\right)^{G}=\left(\xi \tau_{1}\right)^{G}+\cdots+\left(\xi \tau_{p}\right)^{G}$, and thus $\eta(\chi \psi)=p$. We conclude that such $T$ cannot exist and so $\mathbf{Z}(\chi)=\mathbf{Z}(\psi)$.

Given any $z \in \mathrm{Z}(\chi)$ and $g \in G$, we have $z^{g} \cong z(\bmod \operatorname{Ker}(\chi))$ since $Z(G / \operatorname{Ker}(\chi))=$ $\mathrm{Z}(\chi) / \operatorname{Ker}(\chi)$. Hence, $[z, g]=z^{-1} z^{g}$ lies in $\operatorname{Ker}(\chi)$. This same $z$ lies in $\mathrm{Z}(\psi)=\mathrm{Z}(\chi)$. Hence, $[z, g]$ also lies in $\operatorname{Ker}(\psi)$. Therefore, $[z, g] \in \operatorname{Ker}(\chi) \cap \operatorname{Ker}(\psi)=1$ for every $z \in \mathrm{Z}(\chi)=\mathrm{Z}(\psi)$ and every $g \in G$. This implies that $\mathrm{Z}(\chi)=\mathrm{Z}(\psi)=\mathrm{Z}(G)$.

Set $Z=\mathbf{Z}(G)$. Since $Z$ is the centre of $G, \xi^{G}=\chi$ and $\tau^{G}=\psi$, we have

$$
\chi_{Z}=p \xi_{Z} \text { and } \psi_{Z}=p \tau_{Z}
$$

Because $\chi_{Z} \psi_{Z}=p^{2} \xi_{Z} \tau_{Z},(2.4)$ implies that

$$
\left(\theta_{i}\right)_{Z}=p \xi_{Z} \tau_{Z}
$$

for all $i=1, \ldots, n$.

Let $Y / Z$ be a chief factor of $G$ with $Y \leq H$. Since $Z$ is the centre of $G$ and $Z=\mathbf{Z}(\chi)$, the set $\operatorname{Lin}\left(Y \mid \xi_{Z}\right)$ of all extensions of $\xi_{Z}$ to linear characters is $\left\{\left(\xi_{1}\right)_{Y}=\right.$ $\left.\xi_{Y},\left(\xi_{2}\right)_{Y}, \ldots,\left(\xi_{p}\right)_{Y}\right\}$ and it is a single $G$-conjugacy class. By Clifford theory, we have 
that

$$
\chi_{Y}=\sum_{i=1}^{p}\left(\xi_{i}\right)_{Y}
$$

Since $H$ is the stabilizer of $\tau_{Y}$ in $G$ and $\psi(1)=p$, as before we have that the set $\operatorname{Lin}\left(Y \mid \tau_{z}\right)=\left\{\left(\tau_{1}\right)_{Y}=\tau_{Y},\left(\tau_{2}\right)_{Y}, \ldots,\left(\tau_{p}\right)_{Y}\right\}$ and

$$
\psi_{Y}=\sum_{i=1}^{p}\left(\tau_{i}\right)_{Y}
$$

Claim 2.13. The stabilizer $G_{\xi_{Y} \tau_{Y}}=\left\{g \in G \mid\left(\xi_{Y} \tau_{Y}\right)^{g}=\xi_{Y} \tau_{Y}\right\}$ of $\xi_{Y} \tau_{Y} \in \operatorname{Lin}(Y)$ in $G$ is $H$.

Proof. Assume notation (2.4). Since $H$ is an abelian subgroup of index $p$ in $G$, we have that $G_{\xi_{Y} \tau_{Y}} \geq H$ and thus either $G_{\xi_{Y} \tau_{Y}}=H$ or $G_{\xi_{Y} \tau_{Y}}=G$. Suppose $\xi_{Y} \tau_{Y}$ is a $G$-invariant character, i.e. $G_{\xi_{Y} \tau_{Y}}=G$. Since $|Y: Z|=p$ and $\xi_{Y} \tau_{Y}$ is an extension of $\xi_{Z} \tau_{Z}$, it follows then that all the extensions of $\xi_{Z} \tau_{Z}$ to $Y$ are $G$-invariant. Thus, by (2.4) and (2.10), given any $i$, there exists some extension $v_{i} \in \operatorname{Lin}(Y)$ of $\xi_{Z} \tau_{Z}$ such that $\left(\theta_{i}\right)_{Y}=p v_{i}$. Thus, $(\chi \psi)_{Y}=\left(\sum_{i=1}^{n} m_{i} \theta_{i}\right)_{Y}=\sum_{i=1}^{n} m_{i}\left(v_{i}\right)_{Y}=\sum_{i=1}^{n} m_{i} p v_{i}$ has at most $n<\frac{p+1}{2}$ distinct irreducible constituents. On the other hand, by (2.11) and (2.12) we have

$$
(\chi \psi)_{Y}=\chi_{Y} \psi_{Y}=\left(\sum_{i=1}^{p}\left(\xi_{i}\right)_{Y}\right)\left(\sum_{j=1}^{p}\left(\tau_{j}\right)_{Y}\right)=p \sum_{j=1}^{p} \xi_{Y}\left(\tau_{j}\right)_{Y}
$$

and so $(\chi \psi)_{Y}$ has $p$ distinct irreducible constituents. That is a contradiction and thus $G_{\xi_{Y} \tau_{Y}}=H$.

By Clifford theory and the previous claim, we have that for each $i=1, \ldots, n$, there exists a unique character $\sigma_{i} \in \operatorname{Lin}\left(H \mid \xi_{Y} \tau_{Y}\right)$ such that

$$
\theta_{i}=\left(\sigma_{i}\right)^{G}
$$

If $Y=H$, then $|G: Z|=|G: H||H: Z|=p^{2}$. Since $\chi(1)=\psi(1)=p$, by Corollary 2.30 of [5] we have that $\chi$ and $\psi$ vanish outside $Z$. Since $\theta_{i}(1)=p$ for all $i$ and $|G: Z|=\left|G: \mathbf{Z}\left(\theta_{i}\right)\right|=p^{2}$, it follows that there exists a unique irreducible character lying above $\xi_{Z} \tau_{Z}$ and thus $\eta(\chi \psi)=1$.

2.15. Fix a subgroup $X \leq H$ of $G$ such that $X / Y$ is a chief factor of $G$. Let $\alpha$, $\beta \in \operatorname{Lin}(X)$ be the linear characters such that

$$
\alpha=\xi_{X} \text { and } \beta=\tau_{X} .
$$

Since $\sigma_{i}$ lies above $\xi_{Y} \tau_{Y} \in \operatorname{Lin}(Y)$ for all $i$ and $X / Y$ is a chief factor of a $p$-group, there is some $\delta_{i} \in \operatorname{Irr}(X \bmod Y)$ such that

$$
\left(\sigma_{i}\right)_{X}=\delta_{i} \alpha \beta
$$

Claim 2.17. The subgroup $[X, G]$ generates $Y=[X, G] Z$ modulo $Z$. 
Proof. Working with the group $\bar{G}=G / \operatorname{Ker}(\chi)$, using the same argument as in the proof of Claim 3.26 of $[\mathbf{1}]$, we have that $[\bar{X}, \bar{G}]$ generates $\bar{Y}=[\bar{X}, \bar{G}] \bar{Z}$ modulo $\bar{Z}$. Since $Z=\mathbf{Z}(\chi)$, we have that $\operatorname{Ker}(\chi) \leq Z$. Thus, $\bar{Z}=Z / \operatorname{Ker}(\chi)$ and the claim follows.

2.18. Observe that $G / H$ is cyclic of order $p$. So, we may choose $g \in G$ such that the distinct cosets of $H$ in $\mathrm{G}$ are $\mathrm{H}, \mathrm{Hg}, \mathrm{Hg}^{2}, \ldots, \mathrm{Hg}^{\mathrm{p}-1}$.

Since $\chi=\xi^{G}$ and $\xi_{X}=\alpha$, it follows from 2.15 that

$$
\chi_{X}=\alpha+\alpha^{g}+\cdots+\alpha^{g^{p-1}}=\sum_{i=0}^{p-1} \alpha^{g^{i}} .
$$

Similarly, we have that

$$
\psi_{X}=\beta+\beta^{g}+\cdots+\beta^{g^{p-1}}=\sum_{j=0}^{p-1} \beta^{g^{j}} .
$$

Combining the two previous equations, we have that

$$
\chi_{X} \psi_{X}=\left(\sum_{j=0}^{p-1} \alpha^{g^{j}}\right)\left(\sum_{j=0}^{p-1} \beta^{g^{j}}\right)=\sum_{i=0}^{p-1} \sum_{j=0}^{p-1} \alpha^{g^{i}} \beta^{g^{j}} .
$$

By (2.4) and (2.16), we have that

$$
(\chi \psi)_{X}=\left(\sum_{i=1}^{n} m_{i} \theta_{i}\right)_{X}=\sum_{i=1}^{n} m_{i}\left[\sum_{j=0}^{p-1}\left(\delta_{i} \alpha \beta\right)^{g^{j}}\right] .
$$

Claim 2.21. Let $g \in G$ be as in 2.18. For each $i=0,1, \ldots, p-1$, there exist $j \in$ $\{0,1, \ldots, p-1\}$ and $\delta_{g^{i}} \in \operatorname{Lin}(X \bmod Y)$ such that

$$
\alpha \beta^{g^{i}}=(\alpha \beta)^{g^{j}} \delta_{g^{i}}
$$

Also, $\left|\left\{\delta_{g^{i}} \mid i=0,1,2, \ldots, p-1\right\}\right| \leq n$.

Proof. See Proof of Claim 3.30 of [1].

Claim 2.23. Let $g \in G$ be as in 2.18. Then, there exist three distinct integers $i, j, k \in$ $\{0,1,2, \ldots, p-1\}$, and some $\delta \in \operatorname{Irr}(X \bmod Y)$, such that

$$
\alpha \beta^{g^{i}}=(\alpha \beta)^{g^{r}} \delta, \alpha \beta^{g^{j}}=(\alpha \beta)^{g^{s}} \delta \text { and } \alpha \beta^{g^{k}}=(\alpha \beta)^{g^{t}} \delta
$$

for some $r, s, t \in\{0,1,2, \ldots, p-1\}$.

Proof. See Proof of Claim 3.34 of [1].

Claim 2.24. We can choose the element $g$ in 2.18 such that one of the following holds:

(i) There exists some $j=2, \ldots, p-1$ such that

$$
\alpha \beta^{g}=(\alpha \beta)^{g^{r}} \text { and } \alpha \beta^{g^{j}}=(\alpha \beta)^{g^{s}},
$$

for some $r, s \in\{0,1, \ldots, p-1\}$ with $r \neq 1$. 
(ii) There exist $j$ and $k$ such that $1<j<k<p$, and

$$
\alpha \beta^{g}=(\alpha \beta)^{g^{r}} \delta, \alpha \beta^{g^{j}}=(\alpha \beta)^{g^{s}} \delta \text { and } \alpha \beta^{g^{k}}=(\alpha \beta)^{g^{t}} \delta,
$$

for some $\delta \in \operatorname{Irr}(X \bmod Y)$ and some $r, s, t \in\{0,1, \ldots, p-1\}$ with $r \neq 1$.

Proof. See Proof of Claim 3.35 of [1].

Let $g$ be as in Claim 2.24. Since $X / Y$ is cyclic of order $p$, we may choose $x \in X$ such that $X=Y<x>$. Since $H$ is abelian, we have $[X, H]=1$. Suppose that $\left[x, g^{-1}\right] \in Z$. Then, $x$ centralizes both $g^{-1}$ and $H$ modulo $Z$. Hence, $x Z \in \mathbf{Z}(G / Z)$ and so $[x, G] \leq Z$. Since $Y / Z$ is a chief section of the $p$-group $G$, we have that $[Y, G] \leq Z$ and so $[<x>$ $Y, G]=[X, G] \leq Z$ which is false by Claim 2.17. Hence $\left[x, g^{-1}\right] \in Y \backslash Z$ and so

$$
Y=Z<y>\text { is generated over } Z \text { by } y=\left[x, g^{-1}\right] \text {. }
$$

Since $[Y, G] \leq Z$, we have that $z=\left[y, g^{-1}\right] \in Z$. If $z=1$, then $G=H<g>$ centralizes $Y=Z<y>$, since $H$ centralizes $Y<X$ by 2.15 , and $G$ centralizes $Z$. This is impossible because $Z=\mathbf{Z}(G)<Y$. Thus,

$$
z=\left[y, g^{-1}\right] \text { is a non-trivial element of } Z .
$$

By (2.25), we have $y=\left[x, g^{-1}\right]=x^{-1} x^{g^{-1}}$. By (2.26), we have $z=\left[y, g^{-1}\right]=y^{-1} y^{g^{-1}}$. Finally, $z^{g^{-1}}=z$ since $z \in Z$. Since $X=Z<x, y>\leq H$ is abelian, it follows that

$$
z^{g^{-j}}=z, y^{g^{-j}}=y z^{j} \text { and } x^{g^{-j}}=x y^{j} z^{\left(\begin{array}{l}
j \\
2
\end{array}\right)},
$$

for any integer $j=0,1, \ldots, p-1$. Because $g^{-p} \in H$ centralizes $X$ by 2.15 , we have

$$
z^{p}=1 \text { and } y^{p} z^{\left(\frac{p}{2}\right)}=1 .
$$

Observe that the statement is true for $p \leq 3$ since then $\frac{p+1}{2} \leq 2$. Thus, we may assume that $p$ is odd. Hence, $p$ divides $\left(\begin{array}{l}p \\ 2\end{array}\right)=\frac{p(p-1)}{2}$ and $z^{\left(\begin{array}{c}p \\ 2\end{array}\right)}=1$. Therefore,

$$
y^{p}=z^{p}=1 .
$$

It follows that $y^{i}, z^{i}$ and $z^{i / 2}$ depend only on the residue of $i$ modulo $p$, for any integer $i \geq 0$.

2.29. Observe that $\operatorname{Ker}\left(\xi_{Z}\right) \cap \operatorname{Ker}\left(\tau_{Z}\right) \leq \operatorname{Ker}(\chi) \cap \operatorname{Ker}(\psi)=1$ implies that $z$ is not in both $\operatorname{Ker}\left(\xi_{Z}\right)$ and $\operatorname{Ker}\left(\tau_{Z}\right)$. Without loss of generality, we may assume that $\tau_{Z}(z) \neq 1$. Since $\beta$ is an extension of $\tau_{Z}$, we may assume that $\beta(z) \neq 1$.

Claim 2.30. $\xi_{Z} \tau_{Z}(z)$ is primitive pth root of unit.

Proof. Suppose that $\left(\xi_{Z} \tau_{Z}\right)(z)=1$. Then, $\left(\xi_{Z} \tau_{Z}\right)\left(\left[y, g^{-1}\right]\right)=1$ and so $\left(\xi_{Z} \tau_{Z}\right)^{g}(y)=$ $\left(\xi_{Z} \tau_{Z}\right)(y)$. Since $H$ is abelian, $|G: H|=p, \theta_{i}$ lies above $\xi_{Z} \tau_{Z}$ for all $i$ and $g \in G \backslash H$, it follows that $Y=<y, \mathbf{Z}(G)>$ is contained in $\mathbf{Z}\left(\theta_{i}\right)$. This is contradiction with Claim 2.13. Thus, $\left(\xi_{Z} \tau_{Z}\right)(z) \neq 1$. Since $z$ is of order $p$ and $\xi_{Z} \tau_{Z}$ is a linear character, the claim follows.

Claim 2.31. Suppose that

$$
\alpha \beta^{g}=(\alpha \beta)^{g^{r}} \delta
$$


and

$$
\alpha \beta^{g^{j}}=(\alpha \beta)^{g^{s}} \delta
$$

for some $j \in\{0,1, \ldots, p-1\}, \quad j \neq 1$, some $\delta \in \operatorname{Irr}(X \bmod Y)$ and some $r, s \in$ $\{0,1, \ldots, p-1\}$. Then,

$$
\delta(x)=\beta(z)^{h j(r-1)},
$$

where $2 h \equiv 1 \bmod p$.

Proof. By Claim 2.30 and the same argument as in the proof of Claim 3.40 of [1], the statement follows.

Suppose that Claim 2.24 (ii) holds. Then, by Claim 2.31, we have that $\delta(x)=$ $\beta(z)^{h j(r-1)}$ and $\delta(x)=\beta(z)^{h k(r-1)}$. Since $\beta(z)=\tau_{Z}(z)$ is a primitive $p$ th root of unit by 2.29 , we have that $h j(r-1) \equiv h k(r-1) \bmod p$. Since $r \not \equiv 1 \bmod p$ and $2 h \equiv 1 \bmod p$, we have that $k \equiv j \bmod p$, which is a contradiction. Thus, Claim 2.24 (i) must hold.

We now apply Claim 2.31 with $\delta=1$. Thus, $1=\delta(x)=\beta(z)^{h j(r-1)}$. Therefore, $h j(r-$ $1) \equiv 0 \bmod p$. Since $2 h \equiv 1 \bmod p$, either $j \equiv 0 \bmod p$ or $r-1 \equiv 0 \bmod p$. Neither is possible. That is our final contradiction and Lemma 2.3 is proved.

Proof of Theorem $A$. Since $G$ is a nilpotent group, $G$ is the direct product $G_{1} \times G_{2}$ of its Sylow $p$-subgroup $G_{1}$ and its Hall $p^{\prime}$-subgroup $G_{2}$. We can then write $\chi=\chi_{1} \times \chi_{2}$ and $\psi=\psi_{1} \times \psi_{2}$ for some characters $\chi_{1}, \psi_{1} \in \operatorname{Irr}\left(G_{1}\right)$ and some characters $\chi_{2}, \psi_{2} \in$ $\operatorname{Irr}\left(G_{2}\right)$. Since $\chi(1)=p$, we have that $\chi_{2}(1)=1$ and thus $\chi_{2} \psi_{2} \in \operatorname{Irr}\left(G_{2}\right)$. If $\psi(1) \neq p$, since $\psi(1)$ is a prime number, we have that $\psi_{1}(1)=1$ and thus $\chi_{1} \psi_{1}$ is an irreducible. Therefore, $\chi \psi \in \operatorname{Irr}(G)$ and (iv) holds. We may assume then that $\psi(1)=p$ and thus $\psi_{2}(1)=1$. Then, $\chi_{2} \psi_{2}$ is a linear character and so we may assume that $G$ is a $p$-group.

If $\chi \psi$ has a linear constituent, by Lemmas 2.1 and 2.2, we have that (i) or (ii) holds. So, we may assume that all the irreducible constituents of $\chi \psi$ are of degree at least $p$. If $\chi \psi$ has an irreducible constituent of degree $p^{2}$, then $\chi \psi \in \operatorname{Irr}(G)$ and (iv) holds. We may assume then that all the irreducible constituents of $\chi \psi$ have degree $p$. Since $\chi \psi(1)=p^{2}$, it follows that $\eta(\chi \psi) \leq p$. By Lemma 2.3, we have that either $\eta(\chi \psi)=1$ or $\eta(\chi \psi) \geq \frac{p+1}{2}$, and so (iii) holds.

Examples. Fix a prime $p>2$

(i) Let $E$ be an extraspecial group of order $p^{3}$ and $\phi \in \operatorname{Irr}(E)$ of degree $p$. We can check that the product $\phi \bar{\phi}$ is the sum of all the linear characters of $E$.

(ii) In the proof of Proposition 6.1 of [2], an example is constructed of a $p$-group $G$ and a character $\chi \in \operatorname{Irr}(G)$ such that $\chi \bar{\chi}$ is the sum of $p$ distinct linear characters and of $p-1$ distinct irreducible characters of degree $p$.

(iii) Given an extraspecial group $E$ of order $p^{3}$, where $p>2$, and $\phi \in \operatorname{Irr}(E)$ a character of degree $p$, we can check that $\phi \phi$ is a multiple of an irreducible. In Proposition 6.1 of [1], an example is provided of a $p$-group $G$ and a character $\chi \in \operatorname{Irr}(G)$ such that $\eta(\chi \chi)=\frac{p+1}{2}$. In [6], an example is provided of a $p$-group $P$ and two faithful characters $\delta, \epsilon \in \operatorname{Irr}(P)$ of degree $p$ such that $\eta(\delta \epsilon)=p-1$.

Let $G$ be the wreath product of a cyclic group of order $p^{2}$ with a cyclic group of order $p$. Thus, $G$ has a normal abelian subgroup $N$ of order $\left(p^{2}\right)^{p}$ and index $p$. Let $\lambda \in \operatorname{Lin}(N)$ be a nontrivial character. We can check that $\chi=\lambda^{G}$ and $\psi=\left(\lambda^{2}\right)^{G}$ are irreducible characters of degree $p$ and $\chi \psi$ is the sum of $p$ distinct irreducible characters of degree $p$. 
We wonder if there exists a $p$-group $P$ with characters $\chi, \psi \in \operatorname{Irr}(P)$ of degree $p$ such that $\frac{p+1}{2}<\eta(\chi \psi)<p-1$.

(iv) Let $Q$ be a $p$-group and $\kappa \in \operatorname{Irr}(Q)$ be a character of degree $p$. Set $P=Q \times Q$, $\chi=\kappa \times 1_{G}$ and $\psi=1_{G} \times \kappa$. Observe that $\chi, \psi$ and $\chi \psi$ are irreducible characters of $P$.

Acknowledgements. I would like to thank Professor Everett C. Dade for his suggestions. Also, I thank Irene S. Suarez for her encouragement.

\section{REFERENCES}

1. E. Adan-Bante, Products of characters and finite p-groups, J. Algebra 277 (2004) 236255.

2. E. Adan-Bante, Products of characters and finite p-groups II, Arch. Math. 82(4) (2004), 289-297.

3. E. Adan-Bante, Products of characters and derived length II, J. Group Theory 8 (2005), 453-459.

4. E. Adan-Bante, Restriction of characters and products of characters, Israel J. Math 174(1) (2009), 221-225.

5. I. M. Isaacs, Character theory of finite groups (Academic Press, New York-San Francisco-London, 1976)

6. M. Loukaki and A. Moreto, On the number of constituents of products of characters, Algebra Colloq. 14(2) (2007), 207-208. 